\title{
Enhance the Absorption of Gamma-ray Energy Inside the Tumor Using Gold Nanoparticles and Iodine Particles
}

\author{
V. Apanasevich ${ }^{1}$, V. Avramenko² ${ }^{2}$ P. Lukyanov ${ }^{1,3}$, A. Lagureva $^{1,4, *}$, A. Polkovnikova ${ }^{1}$, K. Lukyanenko $^{4}$, V. \\ Kustov $^{5}$, V.Temchenko ${ }^{5}$, I.Agafonova ${ }^{3}$, I.Pankratov ${ }^{6}$, L.Stebunov ${ }^{6}$, S. Bratskaya ${ }^{2}$ \\ ${ }^{1}$ Pacific State Medical University, Vladivostok, 690002, Russia \\ ${ }^{2}$ Institute of Chemistry FEB RAS, Vladivostok, 690022, Russia \\ ${ }^{3}$ G.B.Elyakov Pacific Institute of Bioorganic Chemistry FEB RAS, Vladivostok, 690022, Russia \\ ${ }^{4}$ Far East Federal University, Vladivostok, 690000, Russia \\ ${ }^{5}$ Vladivostok's Branch of the Customs Academy, Vladivostok, 690000, Russia \\ ${ }^{6}$ Primorski Oncology Center, Vladivostok, 690000, Russia \\ *Corresponding Author: sandy767@mail.ru
}

Copyright $(2014$ Horizon Research Publishing All rights reserved.

\begin{abstract}
The problem of searching ways to enhance efficiency of cancer treatment is extremely important. One of the currently recognized methods of treatment is radiation therapy. However, efficiency of radiotherapy is limited by radiosensitivity of intact tissues surrounding the tumor on the one hand, and by radioresistance of the malignancy on the other hand. The problem of overcoming radiation resistance of tumors is a key element in the local control of tumor growth [1]. Introduction of radiosensitizers into a tumor with subsequent irradiation results in secondary radiation inside the tumor, thus increasing the effect of radiotherapy. Various substances capable of converting ionizing radiation can serve as radiosensitizers. This may be diagnostic preparations containing heavy chemical elements, such as iodine, gadolinium, gold nanoparticles, platinum and other chemicals [5]. The aim of our experiment was selection of a drug which would give a significant increase of the secondary radiation in biological tissues when irradiating with gamma rays (GR) with the energy of $1.25 \mathrm{MeV}$. We used as a radiation sensitizer a drug containing gold nanoparticles and iodine particles which, when introduced directly into a tumor, will increase energy of the radiation inside the tumor without increasing the dose permitted according to the plan of exposure.
\end{abstract}

Keywords Tumor, Gold Nanoparticles, Radiosensitivity, Radiomodification, Irradiation, Radiation Therapy

\section{Introduction}

Previous 50 years of the development of radiation therapy have been mostly focused on a search for efficient ways to deliver photons of ionizing radiation into the tumor tissue and to set strict limits for irradiation of surrounding intact tissues. The trends have been reflected by the radiotherapy protocols, namely, IMRT (Intensive Modulation Radio Therapy), IGRT (Image Guided Radio Therapy), SRT (Stereotactic Radio Therapy). The energy of photons used in these protocols ranges from 4 to $20 \mathrm{MeV}$.

Recently, however, the coming therapeutic paradox has become increasingly clear. Photons with the radiation energy in the range of 20 to $200 \mathrm{keV}$ are the most damaging ones, while X-ray penetrating power of the energy is very low. Deep-lying tumors are treated with much higher energy, over $1 \mathrm{MeV}$. The paradox is due to the fact that high-energy photons interact with a matter by creating an electron-positron pair and Compton Effect. In contrast, photons with energies from 20 to $200 \mathrm{keV}$ interact with a matter primarily through the photoelectric effect [2]. Thus, in the process of interaction between photons and atoms which are part of the biological tissue the GR energy undergoes multiple transformations resulting in secondary low energy radiation inside the biological tissue. The low energy radiation has damaging effect on the tissue cells. The probability of such interaction is proportional to mass of the atomic nuclei inside the tissue.

\section{Materials and Methods}

To confirm effectiveness of the experimental introduction of certain drugs in the tumor, we conducted a series of measurements on irradiation of biological material having therein particles of heavy metals. The aim of our experiment was to select a drug which would give a significant increase of the secondary radiation effect in a biological tissue irradiated with gamma rays in the energy range of 20 to $20,000 \mathrm{keV}$. Equipment used for the experiment included gamma-ray spectrometer with a semiconductor detector (purity germanium detector manufactured by ORTEC, crystal dimensions: diameter $46 \mathrm{~mm}$, length $49 \mathrm{~mm}$, the model of the detector GEM-15P4), and two gamma-ray 
sources ${ }^{60} \mathrm{Co}$ (like OSGI) with a total activity about $2 \times 10^{5}$ Bq. Colloidal gold was obtained by thermal reduction of [AuCl4]-complex in alginate solution. Under constant mixing $2.5 \mathrm{ml}$ of $\mathrm{H}[\mathrm{AuCl} 4]$ solution containing $2 \mathrm{mg}$ $(\mathrm{Au}) / \mathrm{mL}$ was added to $50 \mathrm{ml}$ of boiling sodium alginate solution with polymer concentration $0.55 \%$, which was adjusted to $\mathrm{pH} 11$. After 2 minutes solution turned gray, the color change from grayish to wine red was observed during next 20 minutes. The solution was kept boiling under constant mixing for 30 minutes, and then cooled down to room temperature. The volume of solution was adjusted to $50 \mathrm{ml}, \mathrm{pH}$ - to physiological value of 7.4 with addition of $0.1 \mathrm{M} \mathrm{NaOH}$. Maximum of plasmon adsorption was found at $526 \mathrm{~nm}$, the spectrum was recorded using Shimadzu UV-VIS 1650 spectrophotometer. Mean diameter of gold nanoparticles was calculated as $17 \mathrm{~nm}$ using spectral data and taking into account red-shift of plasmon maximum due to the polymer shell around nanoparticle. Final $\mathrm{Au}$ concentration is $100 \mathrm{ug} / \mathrm{ml} .0 .9 \%$ saline solution $(\mathrm{NaCl})$ was used as an analogue of biological tissue. «Ultravist-300» iodine-containing contrast agent for intravascular diagnostic, intrathecal, and intracavitary administration. The irradiated media:

\section{$1.4 \mathrm{~cm}$ of $0.9 \% \mathrm{NaCl}$ solution;}

$2.4 \mathrm{~cm}$ of $0.9 \% \mathrm{NaCl}$ solution $+0.5 \mathrm{~cm}$ of colloidal gold;

$3.4 \mathrm{~cm}$ of $0.9 \% \mathrm{NaCl}$ solution $+0.5 \mathrm{~cm}$ of "Ultravist-300";

$4.4 \mathrm{~cm}$ of $0.9 \% \mathrm{NaCl}$ solution $+0.5 \mathrm{~cm}$ of "Ultravist -300 " + $0.5 \mathrm{~cm}$ colloidal gold.

Energy spectrum of radiation from the radionuclide ${ }^{60} \mathrm{Co}$ source $(1.25 \mathrm{MeV})$ was measured when gamma radiation passing through different combinations of solutions - $\mathrm{NaCl}$, colloidal gold, "Ultravist-300" - placed in a polystyrene Petri dish. Indicators of radiation passing through five empty polystyrene Petri dishes were measured to obtain control values. Nature of the irradiated medium was changed by filling the Petri dishes with test solutions - $\mathrm{NaCl}$, colloidal gold and "Ultravist-300", thickness of the solutions was changed as well. The measurement results were recorded in the form of gamma-spectrometer emission spectra, which varied depending on the solutions placed into the Petri dish. The experimental data were processed by standard statistical methods.

\section{Results and Discussion}

Practical radiation therapy uses x-ray, braking and gamma radiation. X-rays are typically used for radiotherapy of superficial tumors in a human body. Relatively hard gamma radiation is used for irradiating deep seated tumors. The main side effect of radiation therapy of malignant tumors is radiation damage to healthy tissue, and there is a number of ways to minimize the effect. One of such ways is to enhance radio sensitivity of the tumor tissue (tissue radio modification) and thereby to reduce exposure. Radio modification may be realized through introduction of various chemicals - radio sensitizers [3,9].

It is known that the probability of absorption of radiation by atoms increases for atoms with large nuclear charge $Z$ (usually $Z^{4}$ ). This strong dependence allows a suggestion that even low concentration of nanoparticles in the soft tissues will contribute to a significant increase in radiation dose absorption [6,7]. Furthermore, nanoparticles are known to have a surface plasma resonance that has a spectral absorption maximum in the tissue transparency at a wavelength of 750-1200 nm, and significantly enhances the ability of soft tissue to absorb radiation in radiotherapy $[4,10]$.

Introduction of radio sensitizers into a tumor with subsequent irradiation results in secondary radiation inside of the tumor, thus increasing the effect of radiotherapy. Various substances capable of converting ionizing radiation can serve as radio sensitizers. This may be diagnostic preparations containing heavy chemical elements, such as iodine, gadolinium, nanoparticles of gold, platinum and other chemicals $[5,8]$.

Some authors suggest that the radio sensitizing mechanism of gold nanoparticles depends to a great extent on the nature of their coating. However, the discrepancy over the radio sensitizing effects of gold nanoparticles may also be attributed to the different dimensions of gold nanoparticles used as well as the type of tumor cell under study [11].

In our experiment we observed the phenomenon of increasing the absorbed energy of gamma-irradiation in the radiation energy range of 20 to $200 \mathrm{keV}$. In the resulting spectra the quantity of pulses with photon energy of 20 to $200 \mathrm{keV}$ was calculated, which numbered (Fig. 1):

(86.5 \pm 2.4 ) thousand pulses for $4 \mathrm{~cm}$ of $0.9 \% \mathrm{NaCl}$ solution; $(89.5 \pm 2.5)$ thousand pulses for $4 \mathrm{~cm}$ of $0.9 \% \mathrm{NaCl}$ solution + $0.5 \mathrm{~cm}$ of colloidal gold;

$(88.0 \pm 2.4)$ thousand pulses for $4 \mathrm{~cm}$ of $0.9 \% \mathrm{NaCl}$ solution + $0.5 \mathrm{~cm}$ of "Ultravist-300"; and

(95.2 \pm 2.7$)$ thousand pulses for $4 \mathrm{~cm}$ of $0.9 \% \mathrm{NaCl}$ solution + $0.5 \mathrm{~cm}$ of "Ultravist- 300 " $+0.5 \mathrm{~cm}$ colloidal gold.

The result of the research was a calculation of the coefficient of increase of absorbed energy (CIAE) in biological tissue, i.e. ratio of the energy absorbed in biological tissue in the presence of gold nanoparticles and heavy iodine particles to the energy absorbed in biological tissue without heavy particles. The calculations showed that in the low energy range from 20 to $200 \mathrm{keV}$ increase of media absorption is over a unity, i.e. there is additional absorption due to increase in low-energy photons output. CIAE values for various combinations of the subject media are given in Table 1.

Analysis of the experimental results showed that there is a tendency to increasing output of low-energy photons below $200 \mathrm{keV}$ in a composite media. For example, it is seen that the spectrum of the radiation passing through the $0.9 \% \mathrm{NaCl}$ solution in combination with gold $(\mathrm{NaCl}+\mathrm{Au})$ has a $12 \%$ higher total energy in the low energy range of the spectrum 
than the control spectrum (No agents). The spectrum after passing $\mathrm{NaCl}$ combined with colloidal gold and "Ultravist-300" $(\mathrm{NaCl}+\mathrm{Au}+\mathrm{I})$ showed maximum 17\% increase in the output of low-energy photons, which may indicate the occurrence of additional radiation in composite medium containing $\mathrm{NaCl}$, gold nanoparticles and iodine, as a result of low energy photons appearance. In turn, increase of energy absorption by a medium containing only $\mathrm{NaCl}(\mathrm{NaCl})$, as well as by a medium containing $\mathrm{NaCl}$ and "Ultravist-300" $(\mathrm{NaCl}+\mathrm{I})$, is not more than $10 \%$, and is almost the same.

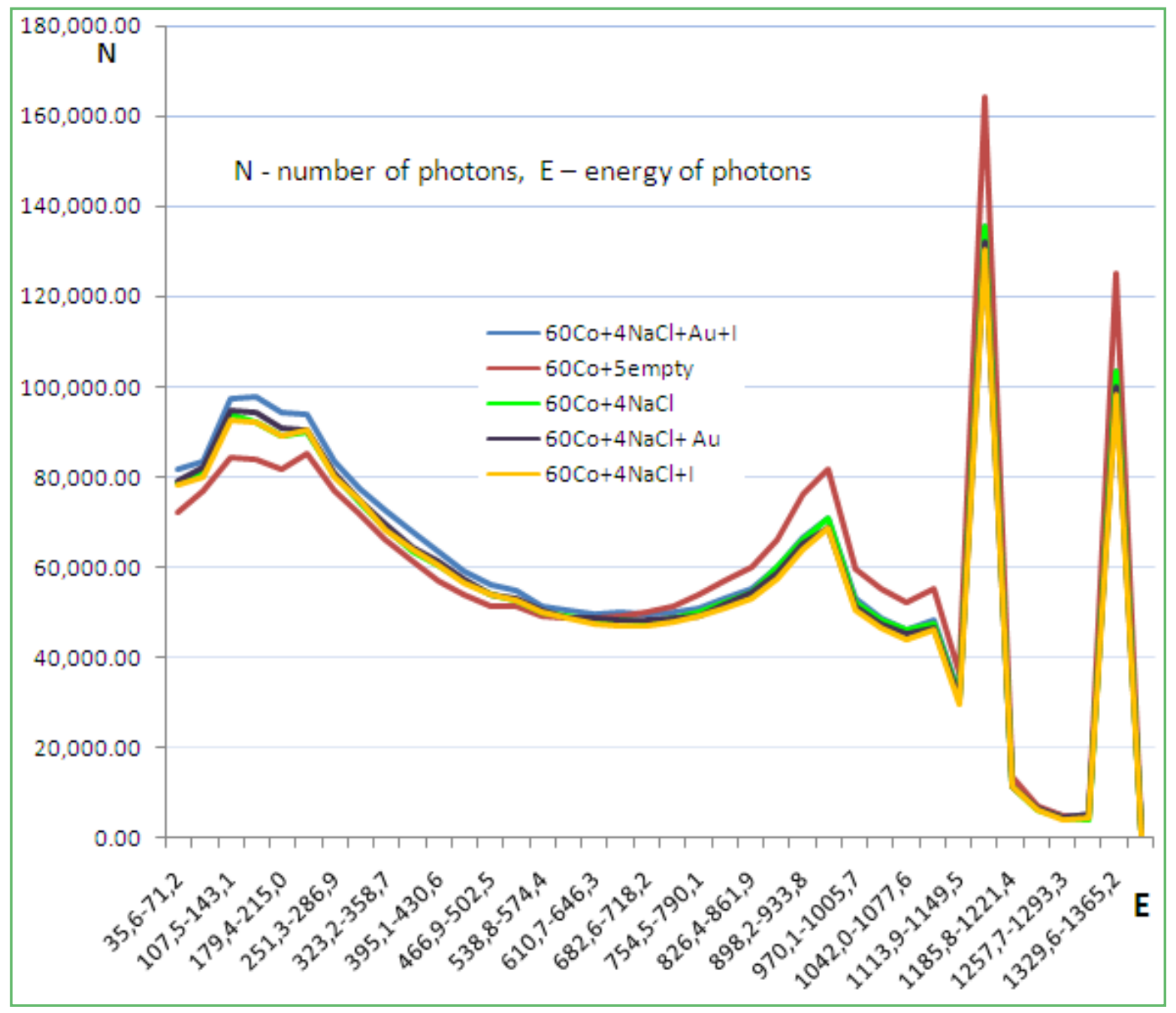

Figure 1. The spectra of absorbed energy of gamma-irradiation.

Table 1. Coefficient of increase of absorbed energy (CIAE) for different media

\begin{tabular}{|c|c|c|c|c|c|}
\hline \multirow{2}{*}{ Energy, $\mathrm{keV}$} & \multicolumn{5}{|c|}{ CIAE for different media, $\%$} \\
\cline { 2 - 6 } & $\mathrm{NaCl}+\mathrm{Au}+\mathrm{I}$ & $\mathrm{NaCl}$ & $\mathrm{NaCl}+\mathrm{Au}$ & $\mathrm{NaCl}+\mathrm{I}$ & No agents \\
\hline $20-70$ & 113 & 109 & 110 & 108 & 100 \\
\hline $71-110$ & 109 & 106 & 107 & 104 & 100 \\
\hline $111-145$ & 115 & 111 & 112 & 110 & 100 \\
\hline $146-180$ & 117 & 110 & 112 & 110 & 100 \\
\hline $181-220$ & 115 & 109 & 111 & 109 & 100 \\
\hline
\end{tabular}




\section{Conclusion}

The presence of a combination of gold nanoparticles and iodine particles in biological material while gamma irradiation increases energy absorption by the biological tissue in the low energy range from $20 \mathrm{keV}$ to $200 \mathrm{keV}$, probably due to increased output of low-energy photons. From therapeutic point of view, heavy particles of gold and iodine emerging on the way of gamma-ray results in formation of a larger number of secondary low-energy photons, which are destructive for tumor cells.

\section{Acknowledgements}

The work is executed with financial support by FEB RAS (Grant № 12-I-P24-17).

\section{REFERENCES}

[1] Jie Gao, Si-ShenFeng, Yajun Guo (2012) Nanomedicine against multidrug resistance in cancer treatment. Nanomedicine, 7, 465-468.

[2] Walter Fendt (2000) The photoelectric effects, February 20,http://www.walter-fendt.de/ph14e/photoeffect.htm.

[3] K. Kimlin, J. Mitchell and R.T. Knight. (2006) Effects of iodinated contrast media on radiation therapy dosimetry for pathologies within the thorax. Australian Institute of Radiography. The Radiographer, 53, 30-34.
[4] S. Jain, D.G.Hirst, J.M. O’Sullivan. (2012) Gold nanoparticles as novel agents for cancer therapy. The British Journal of Radiology, 85, $101-113$.

[5] Wan Nordiana Rahman, NourBishara, Trevor Ackerly, Cheng $\mathrm{Fa} \mathrm{He}$, Price Jackson, Christopher Wong, Robert Davidson, Moshi Geso(2009) Enhancement of radiation effects by gold nanoparticles for superficial radiation therapy Nanomedicine: Nanotechnology, Biology, and Medicine, 5, 136-142.

[6] I.V. Schegolkov, I.N. Sheyno, V.F. Hohlov, A.A. Lipengolts(2010) Simulation of the absorbed dose distributions using Monte Carlo photon technology capture therapy. Medical physics, No. 4, 12-16(Rus.).

[7] Dolgopolov M.A., I.V. Kopytin. (2010)The effect of amplification of radiation exposure in cancer therapy using nanoparticles. Voronezh State University Vestnik, Series: Physics. Mathematics.No. 1, 34-42 (Rus.)

[8] Yuri P.Meshalkin, Natalia P. Bgatova. (2008) The prospects and problems of the use of inorganic nanoparticles in oncology (Review). Journal of Siberian Federal University. Biology, 3, 248-268.

[9] V. Hohlov, V. Kulakov, I. Sheyno, T. Nasonova, V. Mitin, O. Dobrynina. Method «Way of photon capture therapy of tumors», patent RF №2270045, publ., 20.02.2006, C1,

[10] Garif Akchurin, Georgy Akchurin, I.Maksimova, G. Terentyuk, B. Khlebtsov, N. Khlebtsov, V. Tuchin. Method «Way of laser photothermolysis of cancer cells», patent RF №2424831 operates with 22.12.2009

[11] Zhao-Zhin Joanna LIM, Jia-En Jasmine LI, Cheng-Teng NG, Lin-Yue Lanry Yung, Boon-Huat Bay. (2011) Gold nanoparticles in cancer therapy. Acta Pharmacologica Silica, $32,983-990$. 